\title{
Engineering the reactivity of metal catalysts: a model study of methane dehydrogenation on $\mathrm{Rh}(111)$
}

\author{
Anton Kokalj, ${ }^{1,2}$ Nicola Bonini, ${ }^{1}$ Carlo Sbraccia, ${ }^{1}$ Stefano de Gironcoli, ${ }^{1}$ and Stefano Baroni ${ }^{1}$
}

${ }^{1}$ SISSA - Scuola Internazionale Superiore di Studi Avanzati and INFM DEMOCRITOS National

Simulation Center, Via Beirut 2-4, 34014 Trieste, Italy

${ }^{2}$ Jožef Stefan Institute, Jamova 39, SI-1000 Ljubljana, Slovenia

Email: tone.kokalj@ijs.si

RECEIVED DATE (to be automatically inserted after your manuscript is accepted if required according to the journal that you are submitting your paper to)

\section{SUPPORTING INFORMATION}

\section{Computational details}

The calculations were performed in the framework of density-functional theory using the generalized gradient approximation (GGA) of Perdew-Burke-Ernzerhof (PBE). ${ }^{1}$ We have used ultrasoft pseudopotentials ${ }^{2,3}$ and a plane-wave basis set up to a kinetic energy cut-off of 27 Ry (216 Ry for the charge-density cut-off). Brillouin zone (BZ) integrations have been performed with the Gaussianspreading special-point technique ${ }^{4,5}$ and a smearing parameter of $0.03 \mathrm{Ry}$. All the calculations have been performed using the PWscf package, ${ }^{6}$ while molecular graphics has been generated with the XCRYSDEN package. ${ }^{7}$

Surfaces were modeled by periodic super-cells. For the perfect $\mathrm{Rh}(111)$ a slab consisting of four (111) layers was used and molecules were adsorbed on one side of the slab forming a $(2 \times 2)$ surface lattice. The ad-row (ad-atom) defects were modeled by the above slab model and adding onto the fcc sites two (one) additional $\mathrm{Rh}$ atoms per supercell. Hence, the supercells consist of 16, 17, and $18 \mathrm{Rh}$ atoms for the perfect $\mathrm{Rh}(111)$, ad-atom, and ad-row defects, respectively. The step edge defect was modeled by a Rh (211) slab composed of twelve (211) layers, where a $(2 \times 1)$ supercell was used to describe the adsorption phenomena (this model comprises $24 \mathrm{Rh}$ atoms in the supercell). The thickness of the slabs (i.e. number of layers) is sufficient to yield converged results for adsorption energies and activation barriers. For example, 3-, 4-, and 5-layers models of perfect (111) surface give almost the same adsorption energies of methyl $\left(\mathrm{CH}_{3}\right)$ and the activation energy for the $\mathrm{CH}_{4} \rightarrow \mathrm{CH}_{3}+\mathrm{H}$ reaction.

We used the calculated bulk lattice parameter of $3.86 \AA$ as the in-plane lattice spacing. For the perfect surface, ad-row and ad-atom defects only the bottom substrate layer of the slab was fixed, while for the step edge the bottom three (211) layers were fixed. The remaining degrees of freedom were optimized. The thickness of the vacuum (the distance between the molecule and the adjacent slab) was set to about 16 a.u. For the perfect surface, ad-row, and ad-atom defects the BZ integrations were performed with a $4 \times 4$ uniform and shifted $\mathrm{k}$-mesh, ${ }^{8}$ while for the step edge the $4 \times 3 \mathrm{k}$-mesh was used.

Reaction minimum energy paths (MEP) of dehydrogenation reactions were calculated using the nudged elastic band (NEB) method. ${ }^{9}$ To obtain a reliable estimates of activation energies, and transition state structures, a climbing image variant of NEB was applied. ${ }^{10}$ 
In the calculation of the transition state (TS) and a minimum energy path (MEP) using the NEB method, one chooses an initial state (IS) structure (reactants) and a final state (FS) structure (products), and connects the two structures with several images which are interconnected by an elastic string (for an example see Figure S1). It usually happens that several reaction channels exists between a given reactants and products. In the communication we discuss only the best (characterized by the minimum activation energy) obtained MEPs.

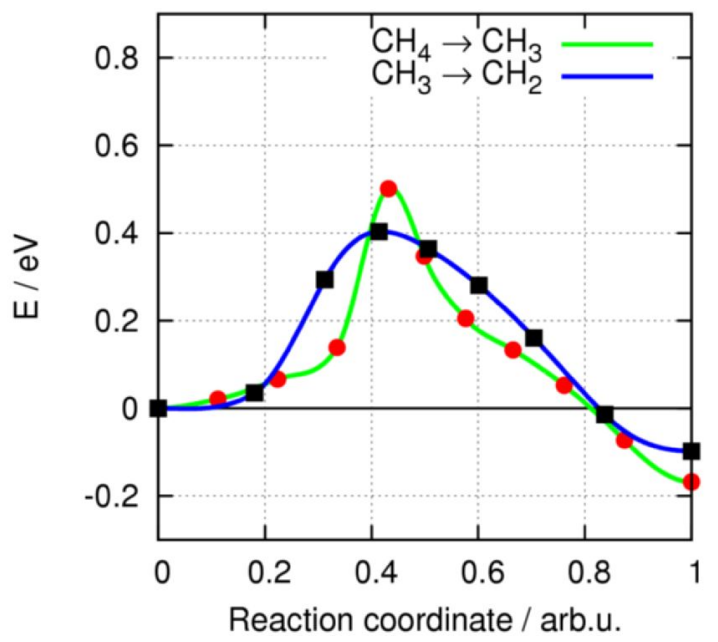

Figure S1. Reaction minimum energy paths for the first two steps of methane dehydrogenation over the (100)-type step edge on the $\mathrm{Rh}(111)$ surface.

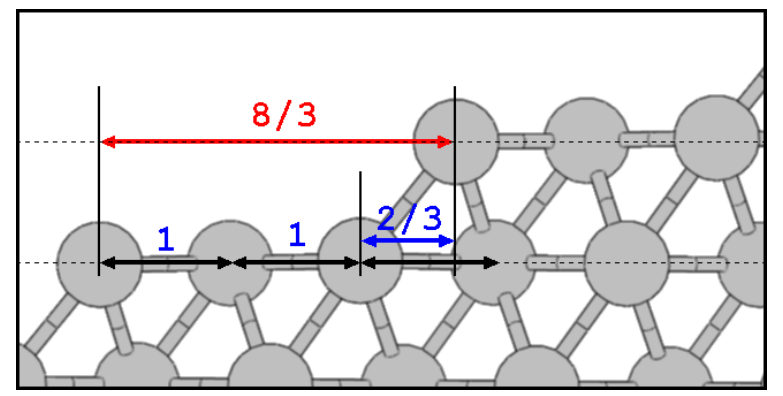

Figure S2. The side view on the fcc (211) vicinal surface. Note that the exposed number of terrace atoms is not 3 but only $2+\frac{2}{3}$, because the inner terrace atom (i.e the one below the step-edge atom) is covered by its neighboring step-edge atom.

\section{Step, ad-row, and ad-atom defects formation energies}

In Table S1 we report the surface energies of the above described models for the perfect (111), stepped (211), ad-row, and ad-atom defects. The formation energies for the step, ad-row, and ad-atom are reported as well. The surface energies of the latter three defected surfaces are higher than that of (111) surface, a fact related to the lower coordinated atoms at defects. Namely, an energy is required for the formation of defects. Indeed, the excess step formation energy can be evaluated using the relation:

$$
\gamma^{\text {step }}=\sum^{\text {step }}-\tilde{n} \sigma^{(111)}
$$

where $\Sigma^{\text {step }}$ is a surface energy per terrace for stepped surface, $\tilde{n}$ is the effective number of atoms in the terrace per $(1 \times 1)$ unit cell, while $\sigma^{(111)}$ is the surface energy per atom for perfect (111) surface. Although 
the (111) terrace of (211) vicinal surface is composed of 3 atoms per $(1 \times 1)$ unit cell, the effective number of atoms $\tilde{n}$ (i.e. the number of exposed terrace atoms) is only $2+\frac{2}{3}$. The number $\tilde{n}$ is shown graphically in Figure S2. For more elaborate discussion on the step formation energies refer to, for example, Ref. 11.

The ad-row and ad-atom formation energy can be evaluated using the relation:

$$
\gamma^{\text {defect }}=\frac{1}{n_{d}} \quad\left\{\Sigma^{\text {defect }}-\Sigma^{(111)}\right\}
$$

where $\Sigma^{\text {defect }}$ is a surface energy per supercell of defected surface, while $\Sigma^{(111)}$ is the surface energy per supercell for perfect (111) surface. The $n_{d}$ is the number of atoms constituting the defect per supercell. For the ad-row the $n_{d}$ is 2 , while for the ad-atom $n_{d}$ is 1 .

Table S1. Calculated surface energies $(\sigma)$, and defect formation energies $(\gamma)$ for four models described in the text.

\begin{tabular}{lcc}
\hline Structure & $\begin{array}{c}\sigma \\
\left(\mathrm{eV} / \AA^{2}\right)\end{array}$ & $\begin{array}{c}\gamma \\
(\mathrm{eV} / \text { defect-atom })\end{array}$ \\
\hline perfect (111) & 0.13 & - \\
step-edge & 0.15 & 0.46 \\
ad-row & 0.21 & 0.99 \\
ad-atom & 0.19 & 1.64 \\
\hline
\end{tabular}

\section{References.}

(1) Perdew, J. P.; Burke, K.; Ernzerhof, M. Phys. Rev. Lett. 1996, 77, 3865.

(2) Vanderbilt, D. Phys. Rev. B 1990, 41, 7892.

(3) The pseudopotentials employed in this communication can be downloaded from the PWscf's pseudopotential download page: http://www.pwscf.org/pseudo.htm (files: Rh.pbe-nd-rrkjus.UPF, C.pbe-rrkjus.UPF, and H.pberrkjus.UPF).

(4) Monkhorst, H. J.; Pack, J. D. Phys. Rev. B 1976, 13, 5188.

(5) Methfessel, M.; Paxton, A. T. Phys. Rev. B 1989, 40, 3616.

(6) Baroni, S.; Dal Corso, A.; de Gironcoli, S.; Giannozzi, P. "PWSCF and PHONON: Plane-Wave PseudoPotential Codes", http://www.pwscf.org/, 2001.

(7) Kokalj, A. J. Mol. Graphics Modelling 1999, 17, 176. Code available from: http://www.xcrysden.org/.

(8) Bonini, N.; Kokalj, A.; Dal Corso, A.; de Gironcoli, S.; Baroni, S. Phys. Rev. B 2004, 69,.

(9) Henkelman, G.; Jonsson, H. J. Chem. Phys. 2000, 113, 9978.

(10) Henkelman, G.; Uberuaga, B. P.; Jonsson, H. J. Chem. Phys. 2000, 113, 9901.

(11) Vitos, L; Skriver, H. L.; Kollár, J. Surf. Sci. 1999, 425, 212. 